\title{
Glycerol Preserved Amnion: A Viable Source of Biological Dressing for Superficial Partial Thickness Facial Burns
}

\author{
Muhammad Sheraz Raza, Muhammad Umar Asif, Zain Ul Abidin, Farrukh Aslam Khalid, Asma Ilyas and Moazzam \\ Nazeer Tarar
}

Department of Plastic Surgery, Jinnah Burn and Reconstructive Surgery Center, Lahore, Pakistan

\begin{abstract}
Objectives: To evaluate the role of amnion as a biological dressing in superficial partial thickness facial burns.

Study design: Randomized controlled trial.

Place and duration of study: Jinnah Burn and Reconstructive Surgery Centre, Lahore, from January 2017 to December 2018.

Methods: Sixty two patients with superficial partial thickness facial burns were randomly divided into two groups (A and B). Ointment containing Polymyxin B and Bacitracin was applied in group $A(n=28)$ while amnion was applied in group $B$ ( $n=34)$. The patients were monitored for the pain related to burns needing analgesia, time of healing of burns, and cosmetic outcome of the facial scarring over a period of six months.

Results: The mean age of the patients was $26.5 \pm 12.2$ years. 39 (62.9\%) patients were male and $23(37.1 \%)$ were females. The mean percent total facial surface area burned was $2.7 \% \pm 1$. None of the patients developed facial wound infections. The average healing time was $6 \pm 2$ days. The mean frequency of analgesia for 3 days of group B was less as compared to group A. infection rate was similar in both groups. No significant difference was observed in VAS score at 1, 3 and 6 month follow up between both groups. Conclusion: Amnion is a safe, cost effective and most readily available biological dressing for superficial partial thickness facial burn management.
\end{abstract}

Key Words: Amnion, Facial burn, Biological dressing, Scarring, Partial thickness burn.

How to cite this article: Raza MS, Asif MU, Abidin ZU, Khalid FA, llyas A, Tarar MN. Glycerol Preserved Amnion: A Viable Source of Biological Dressing for Superficial Partial Thickness Facial Burns. J Coll Physicians Surg Pak 2020; 30(04):394-398. DOI: https://doi.org/10.29271/jcpsp.2020.04.394.

\section{INTRODUCTION}

Burn is a devastating emergency associated with seriously high rates of disabilities, mortality and morbidity. ${ }^{1}$ Scarring is a major issue in survivors, producing major physical and psychological effects in these patients. ${ }^{2}$ Burn injury anywhere on the body can affect a person's life for a considerably long period of time but if the face is involved in the burn process then it seriously scars the patients for life physically and emotionally. For this very reason, burns of face, hands and feet are considered as special burns and managed on priority basis. The main goal in facial burn treatment should be the restoration of normal facial features, acceptable anatomical balance and symmetry and facial expressions. ${ }^{3}$

In the past there have been efforts for treatment of burn wounds with medications having less adverse effects, such as herbal medicines. ${ }^{4,5}$ Human amniotic membrane has been used for centuries as a biological dressing. In China and Japan, amnion is considered a potent medication for the treatment of many diseases. ${ }^{6}$ In Western medicine, amniotic membranes have been used since the beginning of the last century.

Correspondence to: Dr. Zain Ul Abidin, Department of

Plastic Surgery, Jinnah Burn and Reconstructive Surgery

Center, Lahore, Pakistan

E-mail:drzain.abidin@gmail.com

Received: December 30, 2019; Revised: April 04, 2020;

Accepted: April 11, 2020

DOI: https://doi.org/10.29271/jcpsp.2020.04.394
The first reported use of amnion in burn wounds was by Sabella, shortly after Davis used amniotic membrane in skin transplantations in $1910 .^{7}$ However, later it became clear that amnion is a temporary biological dressing not permanent skin alternative. ${ }^{8}$

Amnion based hydrogel and powder application of these products resulted in the most rapid wound closure rates driven primarily by new epithelialization, less contraction, and without immune rejection. ${ }^{9}$ Amniotic membrane combined with the carrier aloe vera in gel format is easy to produce and to apply and has high efficacy as a burn wound healing product. ${ }^{10}$ As a temporary biological dressing, amnion seems effective in pain relief, decrease infection rate, accelerate epithelization ${ }^{11}$ and ease in dressing of wound. ${ }^{12}$ Occlusive dressings or skin substitutes have also been developed in recent years with qualities of reducing pain, reducing fluid loss, providing better infection control and accelerating wound. ${ }^{13}$ Dehydrated human amnion membrane proved to decrease downstream costs such as management of prolonged pain from donor-site morbidity, revision surgeries from scar and contractures of split thickness grafts. ${ }^{14}$ However facial contours and movements have limited their use. Lastly topical antibiotics are used commonly, this is partly due to cost and difficulty of using other modalities on facial burns. ${ }^{15}$

The aim of this study was to assess the potential benefit of the use of layering amnion as biological dressing in reducing the pain and achieving early healing time in partial thickness burns of face. 
Table I: Comparison of TBSA\%, healing time, patient satisfaction score and frequency of analgesia between both groups.

\begin{tabular}{|c|c|c|c|c|c|c|}
\hline & Groups & Mean \pm SD & Median (IQR) & Minimum & Maximum & p-value \\
\hline \multirow{2}{*}{ TBSA\% } & Group A & $35.6 \pm 9.1$ & $36.5(29.3-40.8)$ & 15 & 55 & \multirow{2}{*}{$0.791^{\mathrm{a}}$} \\
\hline & Group B & $34.9 \pm 10.9$ & $34.0(29.0-44.3)$ & 15 & 57 & \\
\hline \multirow{2}{*}{ Healing time (days) } & Group A & $6.2 \pm 0.8$ & $6.0(6.0-7.0)$ & 5 & 8 & \multirow{2}{*}{$0.010^{* b}$} \\
\hline & Group B & $5.7 \pm 0.7$ & $6.0(5.0-6.0)$ & 5 & 7 & \\
\hline \multirow{2}{*}{ PS (1month) } & Group A & $4.2 \pm 1.0$ & $4.0(3.3-5.0)$ & 2 & 6 & \multirow{2}{*}{$0.610^{\mathrm{b}}$} \\
\hline & Group B & $4.1 \pm 1.0$ & $4.0(3.0-5.0)$ & 2 & 6 & \\
\hline \multirow{2}{*}{3 month } & Group A & $4.4 \pm 0.7$ & $4.0(4.0-5.0)$ & 3 & 6 & \multirow{2}{*}{$0.001^{* b}$} \\
\hline & Group B & $5.2 \pm 0.8$ & $5.0(4.8-6.0)$ & 4 & 7 & \\
\hline \multirow{2}{*}{6 month } & Group A & $5.6 \pm 0.6$ & $5.0(5.0-6.0)$ & 4 & 7 & \multirow{2}{*}{$0.003^{* b}$} \\
\hline & Group B & $6.0 \pm 0.6$ & $6.0(6.0-6.0)$ & 5 & 7 & \\
\hline \multirow{2}{*}{6 month } & Group A & $5.6 \pm 1.0$ & $5.5(5.0-6.0)$ & 4 & 7 & \multirow{2}{*}{$0.002^{* b}$} \\
\hline & Group B & $4.8 \pm 0.8$ & $5.0(4.0-5.0)$ & 3 & 6 & \\
\hline
\end{tabular}

Table II: Comparison of patient satisfaction score between both groups according to surgeon.

\begin{tabular}{|c|c|c|c|c|c|c|}
\hline Surgeon VAS score & Groups & Mean \pm SD & Median (IQR) & Minimum & Maximum & $p$-value ${ }^{b}$ \\
\hline \multirow{2}{*}{ PS (1month) } & Group A & $6.4 \pm 0.6$ & $6.0(6.0-7.0)$ & 5 & 7 & \multirow{2}{*}{0.942} \\
\hline & Group B & $6.4 \pm 0.6$ & $6.0(6.0-7.0)$ & 5 & 7 & \\
\hline \multirow{2}{*}{3 month } & Group A & $5.8 \pm 0.9$ & $6.0(5.0-6.7)$ & 4 & 7 & \multirow{2}{*}{0.494} \\
\hline & Group B & $5.9 \pm 0.9$ & $6.0(6.0-7.0)$ & 4 & 7 & \\
\hline \multirow{2}{*}{6 month } & Group A & $6.4 \pm 0.8$ & $6.0(6.0-7.0)$ & 4 & 8 & \multirow{2}{*}{0.410} \\
\hline & Group B & $6.6 \pm 0.9$ & $6.5(6.0-7.0)$ & 4 & 8 & \\
\hline
\end{tabular}

\section{METHODOLOGY}

This study was conducted at Jinnah Burn and Reconstructive Surgery Centre, Lahore, between January 2017 and December 2018. After permission from ethical review committee of hospital, patients who fulfilled inclusion criteria were randomly divided into two groups A and B. Group A was the control group while group $B$ was study group. Inclusion criteria include patients between 13 and 60 years of age with no major co morbid conditions. Superficial partial thickness burns confirmed by two separate surgeons with three years of experience. Patients with advanced cardiac or pulmonary diseases, uncontrolled Diabetes mellitus and hypertension, and with concomitant injuries including head trauma were excluded from study.

Informed written consent was taken from all enrolled patients and they were randomized in two groups by numerical method. At presentation, the depth of burn and follow up healing was assessed by two burn surgeons. In all patients presenting in the resusciataion area, face was washed with saline. Contamination and dead skin was removed, affected area was cleaned with dilute povidone iodine solution, dried with sterile gauzes and dressed as follows in each group.

In group $A$, the patients were managed with conventional protocol of applying antiseptic ointment (polymyxin B and Bacitracin). In subsequent period the ointment was not completely removed while washing but reinforced with further ointment when face appeared dry. In group B, amnion obtained from elective caesarean sections, was applied.

Informed consent was taken from all enrolled expecting mothers. Donor mothers were preoperatively tested negative for HCV, HBV and HIV. Generally, healthy mothers were isolated with no systemic disorders or urinary and gynecological tract infections. Placenta from deliveries with history of premature rupture membranes and meconium ileus were discarded. The placenta was transported from Gynecology Theater to burn center in sterile container with normal saline. Amnion was separated carefully from chorion. Stained blood from placenta was cleaned by washing with large volume of normal saline until a smooth, whitish transparent layer remained. Tissue cultures from the membrane were sent. Membranes are then transferred to another container containing 30\% glycerol for $12 \mathrm{hrs}$. at room temperature. Meanwhile the cultures were reviewed and membranes devoid of any growth were stored at $4{ }^{\circ} \mathrm{C}$ in refrigerator, in sterile containers containing $70 \%$ glycerol. Amnion was applied as a facial subunit after soaked for $10 \mathrm{~min}$ in a kidney tray containing normal saline.

Baseline analgesia of injection tramadol intravenously was given to all patients as background analgesia. In case of persistent pain despite background analgesia after confirming that pain was due to facial burn a break through analgesia was administered in the form of inj. morphine $3 \mathrm{mg}$ intravenous. Frequency of break through required was noted for each patient of each group. Patients were assessed in both morning 
and evening rounds for development of infection which might be in the form of cellulitis or purulent discharge. In case of infection the face was thoroughly washed, both antibiotic ointment layers and amnion removed, cultures taken and antibiotic therapy accordingly was administered.
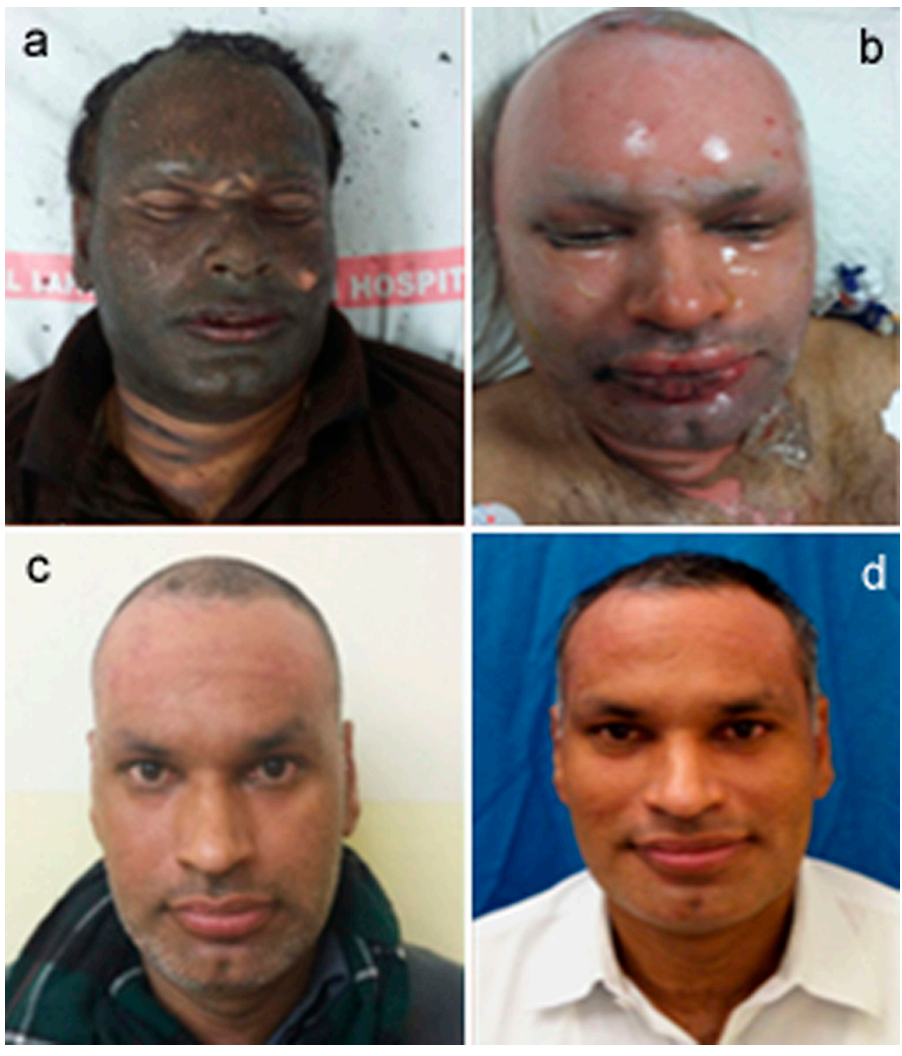

Figure 1: (a) At presentation, (b) amnion applied, (c) after 3 weeks, (d) after 6 weeks.

Healing was defined as when the whole burnt area on the face turned dry with epithelization. Hence, the days taken in each patient for healing were documented. Patients of both groups were assessed at one month, three month and six month interval for Cosmetic outcome by a totally independent set of two Plastic Surgeons who were double blinded, each of whom gave a score out of 10 .

The data were analyzed using SPSS (Statistical Package for Social Sciences) version 23. Frequency and percentage was calculated for gender and infection. Median (IQR), minimum and maximum along with mean and standard deviation was calculated for age, TBSA (Total body surface area)\%, healing time and patient satisfaction score. Shapiro Wilk test was used to check the normality of data. Independent sample t-test and Mann Whitney $U$ test were used to observe mean difference in age, TBSA\%, healing time and patient satisfaction score between both groups. Chi-Square test was applied on the gender variable "along with Fisher's exact test to compare the infection rate between both groups. A p-value $<0.05$ was considered as significant.

\section{RESULTS}

Among the total number of patients, three patients died of concomitant injuries. Two patients of group $A$ and one patient from group B lost to follow up, so 3 more patients were incorporated. Mean TBSA was similar in both groups. The mean healing time of group $B$ was less as compared to group A (Table I). No significant difference was observed at 1 month. But significant difference observed at 3 and 6 months follow up. Mean frequency of analgesia for 3 days in both groups was significant. The mean frequency of analgesia for 3 days of group B was less as compared to group A. infection rate was similar in both groups. No significant difference was observed in Surgeon VAS score at 1, 3 and 6 month follow up between both groups. The mean age was $35.4 \pm 10.7$ years in Group A, and $34.6 \pm 9.9$ years in group $B$. Minimum age in group $A$ was 16 years and maximum was 53 years, similarly 17 years and 51 years in group $B$ patients $(p=0.75)$. Seventeen $(60.7 \%)$ males and 11 (39.3\%) females in group A, while in group B 22 $(64.7 \%)$ were males and $12(35.3 \%)$ were females $(p=0.74)$. Comparison of TBSA\%, healing time, patient satisfaction score and frequency of Analgesia between both groups are given in Table I. Out of the 28 patients in group A, four (14.3\%) developed infection, and 1 (2.9\%) out of 34 got infected. P value of 0.166 was calculated among both groups by applying Fisher's exact test.

\section{DISCUSSION}

An ideal dressing for burns provides relief from pain, protects the wound from secondary infection and maintains a moist environment. Moreover it prevents heat and fluid loss; it should be elastic and non-allergenic and promote healing. Lastly, it should be economical, easily available and easy to apply. ${ }^{16,17}$

Overall the amniotic membrane is composed principally of three types of material: structural collagen and extracellular matrix, biologically active cells and a large number of important regenerative molecules. There are pluripotent stem cells and epithelial cells which are biologically active in the healing process through various receptors on cell membrane. Regenerative biomolecules important in healing and growth process include epidermal growth factors, transforming growth factor (TGF0 Beta, fibroblast derived growth factors, platelet derived growth factors, metalloproteinases and TIMPs (Tissue inhibitors of metalloproteinases), all of which have been found to be abundant in amniotic membrane. ${ }^{18}$ In addition there is lack of HLA-A, HLA-B, HLA-C, making amnion an immunologically privileged material that does not present itself as a foreign body. All these qualities make amnion a near ideal dressing for all kinds of wounds. ${ }^{19}$

There are multiple methods of processing and preserving amnion in literature. These include cryo preservation in liquid nitrogen, preserved in silver nitrate solution and dried irradiated amnion. ${ }^{19-21}$ Dry irradiated and cryo- preserved amnion is costly; similarly silver nitrate preservation does not allow long term preservation. ${ }^{20-22}$ Glycerol-preserved amnion was found up to the mark in terms of use as a biological dressing as it has a balance in getting rid of most of cellular components, making it more and more safe without much potential for transmission of disease, ${ }^{23}$ while retaining enough biological molecules to potentiate healing process. The risk of transmis- 
sion of viral diseases is greatly reduced by screening for HIV, HCV AND HBS antigen, although there is no report of disease transmission through amniotic membrane. ${ }^{24}$ Glycerolpreserved amniotic membrane can be safely used for about 2.5 years. ${ }^{18}$ But a close liaison between gynecology department and burn center is required for preoperative assessment of donor mothers and screening for any communicable disease.

Initially, it was applied as single sheet on forehead and rest of the face avoiding openings. But after drying, it stiffened and restricting the patient regarding facial movements, mastication and mouth opening. So the technique later modified by applying it in facial subunits. No special equipment or skills were required for application which reduces not only patient discomfort, but nursing time as well. All air bubbles and fluid were removed out to achieve complete contact.

The protocol for facial burn cleaning is pretty much common in all centers. The authors used the same protocol of washing with saline and scrubbing with povidone iodine solution. A number of topical applications have been used in facial burns some antiseptic and some antibiotic with the mutual effect of both to provide moist environment for wound healing. Antiseptic applications include alcohol based preparations like chlorhexidine or iodine based preparations like povidone pastes. A comparison of the bactericidal effectiveness of chlorhexidine vs. povidone-iodine preparations failed to show differences in colony count or advantages with the use of either antiseptic. Antimicrobial agent that is most commonly used is Polymyxin B and Bacitracin containing ointment. At the study center, the standard management of facial superficial burns has been application of antimicrobial ointment as well. Its effectiveness has been found to be comparable to a number of other dressings both in preventing infection and promoting healing. Other antimicrobial agents used are silver compounds which have been found to be effective but in exposed areas like face hands and feet it is believed to leave staining that is not very well tolerated.

Other agents like allograft, xenograft and cultured epithelial autograft has been used as biological dressing of face in case of facial burns. They promote healing and have an improved aesthetic outcome but they are not easy to apply on crevices of face and quite expensive as well. Skin substitutes such as dermal engineered skin (Integra), human dermal matrix (alloderm) etc., have also been attempted as dressing and have shown good results but their availability and cost are major issues. $^{24}$

Amnion has been studied as biological dressing on face by Branski et.al. in pediatric age group. They have found it to be more effective dressing compared to antimicrobial ambulant applications, in terms of early healing but final aesthetic outcome of scarring was the same. ${ }^{25}$ In this study, the role of Amnion as dressing for facial burns was studied in adult age group.

Glycerol preserved amnion is one of the most economical dressings. Amnion is washed with saline. One liter of glycerol costs about Rs. 250. The volume of saline used is about 500 $\mathrm{ml}$. Finally, storage requires only an ordinary refrigerator. The economy and the most readily availability, with children being born every hour, makes amnion an extremely suitable dressing in a burn unit.

\section{CONCLUSION}

Most accidental facial burns are superficial and superficial partial thickness, often affecting mainly children. Glycerol preserved amnion is a simple, convenient, economical but effective dressing with a long shelf life. In addition treatment can be offered to outpatient basis as well, helping to reduce the load on hospital beds.

\section{ETHICAL APPROVAL:}

Permissions from the concerned administrative authorities and Ethical Committee were taken.

\section{PATIENTS' CONSENT:}

Informed consent was obtained from patients to publish the data concerning this case.

\section{CONFLICT OF INTEREST:}

Authors have no financial and personal relationships with any organization that could influence this work.

\section{AUTHORS' CONTRIBUTION:}

MSR: Drafted and finalized the manuscript. The author read and approved the final manuscript.

UA: manuscript writing, SPSS data analysis, operated on patients.

FAK: Patient's clinical evaluation, operating surgeon, contri-bution towards results preparation.

ZUA: Final manuscript preparation. Contribution to result analysis and discussion.

Al: Data analysis and manuscript writing.

MNT: Conceptualization of study.

\section{REFERENCES}

1. Pasalar M, Mohammadi AA, Rajaeefard AR, Neghab M, Tolidie $H R$, Mehrabani D. Epidemiology of burns during pregnancy in southern Iran: Effect on maternal and fetal outcomes. World Appl Sci J 2013; 28:153-8.

2. Tanideh N, Haddadi MH, Rokni-Hosseini MH, Hossienzadeh M, Mehrabani D, Sayehmiri K, et al. The healing effect of scrophularia striata on experimental burn wounds infected to pseudomonas aeruginosa in rat. World J Plast Surg 2015; 4:16-23.

3. Tanideh N, Rokhsari P, Mehrabani D, Mohammadi Samani S, Sabet Sarvestani F, Ashraf MJ, et al. The healing effect of licorice on Pseudomonas aeruginosa infected burn wounds in experimental rat model. World J Plast Surg 2014; 3:99-106.

4. Dougherty WR, Spence RJ. Reconstruction of the burned face/cheek: acute and delayed. In: Sood R, Achauer BM, Eds. Achauer and Sood's burn surgery: reconstruction and rehabilitation. Elsevier/Saunders; 2006. p.234-53.

5. Mehrabani D, Farjam M, Geramizadeh B, Tanideh N, Amini M, Panjehshahin MR. The Healing effect of curcumin on burn wounds in rat. World J Plast Surg 2015; 4:29-35.

6. Mohammadi AA, Johari HG, Eskandari S. Effect of amniotic membrane on graft take in extremity burns. Burns 2013; 
39:1137-41.

7. Ullah MS, Ferdous KMN, Mullick MMH, Alam MR, Rahman MS, Khan AR. Amnion membrane graft dressing in superficial partial thickness burn in pediatric patients. Chattagram MaaO-Shishu Hospital Med Coll J 2015; 14:22-5.

8. Mostaque AK, Abdur Rahman KBM. Comparisons of the effects of biological membrane (amnion) and silver sulfadiazine in the management of burn wounds in children. J Burn Care Res 2011; 32:200-9.

9. Murphy SV, Skardal A, Nelson RA Jr, Sunnon K, Reid T, Clouse $C$, et al. Amnion membrane hydrogel and amnion membrane powder accelerate wound healing in a full thickness porcine skin wound model. Stem Cells Transl Med 2020; 9:80-92.

10. Rahman MS, Islam R, Rana MM, Spitzhorn LS, Rahman MS, Adjaye J, et al. Characterization of burn wound healing gel prepared from human amniotic membrane and Aloe vera extract. BMC Complement Altern Med 2019; 19:115.

11. Jeschke MG, Shahrokhi S, Finnerty CC, Branski LK, Dibildox M; ABA Organization \& Delivery of Burn Care Committee. Wound coverage technologies in burn care: Established techniques. J Burn Care Res 2018; 39:313-8

12. Glat PM. Using dehydrated human amnion/chorion membrane allografts in clinical practice. Ann Plast Surg 2017; 78(2 Suppl 1):S1.

13. Demling RH, DeSanti L. Management of partial thickness facial burns (comparison of topical antibiotics and bioengineered skin substitutes). Burns 1999; 25:256-61.

14. Ahuja N, Jin R, Powers C, Bill A, Bass K. Dehydrated human amnion chorion membrane as treatment for pediatric burns. Adv Wound Care 2019.

15. Roper-Hall M. Immediate treatment of thermal and chemical burns. In: Troutman R, Ed. Plastics and reconstructive surgery. Washington: Butterworth; 1962. p. 1991.

16. Mohammadi AA, Mohammadi KM. How Does Human amniotic membrane help major burn patients who need skin grafting: A new experiences? http://www.intechopen.com/predownload/ 189412020.

17. Eskandarlou M, Azimi M, Rabiee S, Seif Rabiee MA. The healing effect of amniotic membrane in burn patients. World J Plast Surg 2016; 5:39-44.

18. Ravishanker R, Bath AS, Roy R. "Amnion Bank" - the use of long term glycerol preserved amniotic membranes in the management of superficial and superficial partial thickness burns. Burns 2003; 29:369-74

19. Branski LK, Herndon DN, Celis MM, Norbury WB, MastersOE, Jeschke MG. Amnion in the treatment of pediatric partial-thickness facial burns. Burns 2008; 34:393-9

20. Bravo D, Rigley TH, Gibran N, Strong M, Newman-Gage H. Effect of storage and preservation methods on viability of transplantable human skin allografts. Burns 2000; 26:367-78

21. Haberal M, Oner Z, Bayraktar U, Bilgin N. The use of silver nitrate incorporated amniotic membranes as a temporary dressing. Burns 1987; 13:159-63.

22. Bujang-Safawi E, Halim AS, Khoo TL, Dorai AA. Dried irradiated human amniotic membrane as a biological dressing for facial burns-A 7-year case series Burns 2010; 36:876-82.

23. Witt JG, Haugh AM, Hauch A, Darden M, Parker G, Ellsworth AW, et al. Amniotic Membrane for the Treatment of Burns. J Plast Surg Cosmetol 2017; 1:1

24. Mohammad AA, Eskandari S, Johari HG, Rajabnejad A. Using amniotic membrane as a novel method to reduce post burn hypertrophic scar formation: A prospective follow-up study. J Cutan Aesthet Surg 2017; 10:13-7.

25. Nouri M, Ebrahimi M, Bagheri T, Fatemi MJ, Najafbeygi A, Araghi $S$, et al. Healing effects of dried and acellular human amniotic membrane and mepitelas for coverage of skin graft donor areas; a randomized clinical trial. Bull Emerg Trauma 2018; 6:195-200. 